Invertebrate Biology 125(1): 34-44.

(C) 2006, The Authors

Journal compilation (C) 2006, The American Microscopical Society, Inc.

DOI: $10.1111 / \mathrm{j} .1744-7410.2006 .00037 . \mathrm{x}$

\title{
In vitro culture of glochidia from the freshwater mussel Anodonta cygnea
}

\author{
Paula Lima, ${ }^{1}$ Uthaiwan Kovitvadhi, ${ }^{2}$ Satit Kovitvadhi, ${ }^{3}$ and Jorge Machado ${ }^{1,4, a}$ \\ ${ }^{1}$ Instituto de Ciências Biomédicas de Abel Salazar, Departamento de Produção Aquática, \\ Universidade do Porto, Porto 4099-003, Portugal \\ ${ }^{2}$ Department of Zoology, Faculty of Science, Kasetsart University, Bangkok 10900, Thailand \\ ${ }^{3}$ Department of Agriculture, Faculty of Science and Technology, Rajabhat Bansomdejchaopraya \\ University, Bangkok 10600, Thailand \\ ${ }^{4}$ CIIMAR — Centro Interdisciplinar de Investigação Marítima e Ambiental, \\ Universidade do Porto, Porto 4050-123, Portugal
}

\begin{abstract}
Larvae of the freshwater swan mussel, Anodonta cygnea, were cultured in artificial media at the controlled temperature of $23^{\circ} \pm 2^{\circ} \mathrm{C}$, with successful metamorphosis for the first time. The artificial medium contained a mixture of M199, common carp plasma, and antibiotics/antimycotics. Glochidia were reared to the juvenile stage in the medium after 10-11 d of culture. After $15 \mathrm{~d}$ of controlled feeding with phytoplankton, the juveniles showed an elongated shell with several growth lines. Larval survival was $34.3 \pm 9.3 \%$, whereas the proportion undergoing metamorphosis was $\leq 60.8 \pm 4.2 \%$. The ultrastructure of early developmental stages was observed by scanning electron microscopy, from the glochidial to the juvenile stage. Glochidia had a hooked shell, with two equal triangular valves formed by a calcareous layer with numerous pores and covered by a thin cuticle of chitin-keratin. The appearance of the complete foot within $11 \mathrm{~d}$ of in vitro culture was considered the final feature of metamorphosis to the juvenile stage. The main alteration during juvenile development was the formation, under the glochidial shell, of a new periostracum with growth lines. The prominent foot, gradually covered by long, dense cilia, showed rhythmical movements involved in the capture of particulate matter. Similarly, cilia and microvilli present in the mantle also performed the same role. Longer cilia, sparsely distributed in the mantle, may function as chemotactile sensors.
\end{abstract}

Additional key words: mussel, metamorphosis, larvae, juveniles, artificial culture

The life cycles of members of the superfamily Unionoidea are atypical among bivalves, because they include a free-living adult and a short-lived obligatory ecto-parasitic larval (glochidia) phase. The larvae of the Unionoidea usually parasitize fish, usually for a period of 10-30 d (Mansur \& Silva 1999). Glochidial metamorphosis depends on fish blood composition and its immune responses, which is the determining factor in mussel-host specificity (Reuling 1919; Arey 1932; Kirk \& Layzer 1997). Chemical or physical contact with the fish may induce glochidial release from the mussel, sometimes in a specific way (Jokela \& Palokangas 1993; Haag \& Warren 1999, 2000). Host specificity is known to be an important factor influencing glochidia infestation and meta-

\footnotetext{
${ }^{a}$ Author for correspondence. E-mail: jmachado@icbas.up.pt
}

morphosis (Haag \& Warren 1999) with respect to specific immunological reactions from the host fish (Meyers \& Millemann 1977; Meyers et al. 1980; O'Connell \& Neves 1999; Rogers et al. 2001). The glochidial phase of development constitutes a critical period because of specific host antibodies to the glochidia (Kirk \& Layzer 1997). Only a small percentage of glochidia reach the juvenile stage because of the low probability of attachment to a host fish (Kirk \& Layzer 1997).

The post-infestation phase begins with juveniles being released from the fish to the substratum, where they are exposed to new hazards (Keller \& Zam 1990; Uthaiwan et al. 2001a,b). A high juvenile mortality in nature is assumed, because of predators, bacteria, protozoa, and fungi. Therefore, the complex process of mimicking fish infestation in the laboratory, where these negative factors can be reduced only partially, has a limited contribution to the maintenance of 
natural mussel populations; however, controlled infestation is still the only practical method for doing so.

It is possible to culture glochidia in an artificial medium, bypassing the parasitic stage and producing free-living juveniles, achieving high production as well as preventing contamination. For these reasons, Isom \& Hudson (1982, 1984a,b), Keller \& Zam (1990), and Uthaiwan et al. (2001a,b, 2002, 2003) developed simple culture techniques for glochidia in artificial media, which resulted in high survivorship. This method may also provide suitable conditions for observing glochidial metamorphosis. According to Uthaiwan et al. (2001a, 2002), the survival and successful metamorphosis of glochidia were clearly increased if M199 medium with carp plasma was used. From the results of Uthaiwan et al. (2001a,b, 2002, 2003), it was possible to improve the rate of glochidial development and juvenile growth in Hyriopsis myersiana LEA 1856 within in vitro culture by applying the specificity principles to the artificial media. In fact, cultures of $H$. myersiana showed high glochidial survival $(93 \%)$ from which successful metamorphosis of the entire culture $(100 \%)$ occurred in artificial culture with plasma of common carp, whereas with Nile tilapia, giant walking catfish, or striped catfish plasma, survival was significantly lower. However, the transformed juveniles only survived for 2 months, suggesting that juvenile survival is a poorly understood process or that the juveniles raised on the artificial medium were not physiologically healthy.

The main goals of this study were to test the viability of artificial culture media for the production of individuals of Anodonta cygnea LinNAeus 1758 and describe morphological alterations during metamorphosis. Therefore, culture techniques of Uthaiwan et al. (2001a) were adapted to $A$. cygnea using plasma from the native common carp (Cyprinus carpio LinNaeus 1758) as well as horse serum for comparative study. The most important steps concern the preparation of glochidia without contamination to produce viable juveniles through successful metamorphosis using an inexpensive culture system. The microscopic morphology associated with glochidial metamorphosis and juvenile growth has not been investigated in A. cygnea under artificial culture conditions. Thus, we report ultrastructural changes by scanning electron microscopy (SEM) and aspects of behavior from glochidium to juvenile.

\section{Methods}

Gravid mussels of Anodonta cygnea were collected, during brooding periods, from Mira Lagoon in Aveiro district, Portugal, and acclimated in 150-L tanks with aeration and mud substratum from the collection site. The photoperiod was roughly $12 \mathrm{~h}$ of light and $12 \mathrm{~h}$ of darkness. The dechlorinated tapwater was kept at room temperature, with a $\mathrm{pH}$ of 7.58.0. The animals were fed a diet of microalgae from support cultures, mimicking microalgal composition in the natural habitat. Gravid females, for glochidia extraction, were chosen on the basis of active ventilation, water ejection, and tight closure of the valves when disturbed.

\section{Preparation of glochidia}

Soil and algae were thoroughly removed from the outer shells of the gravid mussel. Tongs were used to open the shell slightly in order to observe marsupial color, which indicated the stage of embryonic development. The glochidia were examined under a light microscope $(\times 100)$; if their valves periodically closed, they were considered strong and suitable for culturing in artificial media. Viable glochidia were collected by injecting water into the female's marsupial gills to flush glochidia into a beaker filled with water. Thereafter, the water was changed slowly several times to eliminate tissue residues, mucus, and glochidial shell fragments. When there were no more residues, the cleaning was considered complete and the stronger glochidia were used for culture. It is recommended that glochidia from gravid mussels be placed in an artificial medium no later than $5 \mathrm{~h}$ after harvesting, as mortality increases rapidly thereafter (Uthaiwan et al. 2001a).

\section{Glochidial culture}

Glochidia were cultured in artificial medium based on the modifications of Uthaiwan et al. (2001a), which improved the formulae of Isom \& Hudson (1982, 1984a,b) and Keller \& Zam (1990). This medium contained fish plasma (Cyprinus carpio) as a source of protein. The common carp was chosen as the host fish because, although it is a native of Western Europe and East Asia, extensive introductions have helped to make it the world's most widely distributed freshwater fish. In Portugal, it exists in almost every hydrographical basin south of the Douro River.

Glochidia were cultured in tissue culture dishes, each containing $3.5 \mathrm{~mL}$ of artificial medium with fish plasma. In this medium we incubated $\sim 50-100$ glochidia/dish. The natural supply of nutrients, growth factors, oxygen, and $\mathrm{pH}$ maintenance were simulated by artificial conditions. This culture medium was changed after $5 \mathrm{~d}$, because Uthaiwan et al. (2002) 
Table 1. Composition of artificial medium for culture of glochidia.

\begin{tabular}{lc}
\hline \hline Composition of media & $\begin{array}{c}\text { Concentration of } \\
\text { artificial medium }(\mathrm{mL})\end{array}$ \\
\hline M199 & 2.0 \\
Fish plasma & 1.0 \\
Antibiotics and antimycotic* & 0.5 \\
\hline
\end{tabular}

*Details in Table 2.

found that doing so increased glochidial survival. However, this should be done carefully because disturbance caused by manipulation or medium flux can introduce a stress effect during metamorphosis. Additionally, to prevent contamination from symbiotic organisms, antibiotic and antimycotic compounds were added to the medium as summarized in Tables 1 and 2 .

All glochidia in tissue culture dishes were placed inside an incubator with a constant supply of 5\% $\mathrm{CO}_{2}+95 \%$ room air and $50 \%$ humidity. The internal temperature in the incubator was kept at $23^{\circ} \pm 2^{\circ} \mathrm{C}$ until glochidia transformed to the active juvenile stage. Foot extension outside the shell indicated complete transformation. Counts of glochidial mortality were then performed under a light microscope to quantify success of glochidial metamorphosis to the juvenile stage.

\section{Juvenile culture}

Following complete metamorphosis, usually after $10-11 \mathrm{~d}$, early juveniles were removed from the culture medium, rinsed in a mixture of sterilized water and M199, and placed in a beaker containing 200$300 \mathrm{~mL}$ of dechlorinated and aerated water. The juveniles were fed a mixture of three phytoplankton species (Chlamydomonas sp., Monoraphidium sp., and Chlorella sp.), collected from stock cultures and added to the water until a slightly green coloration was

Table 2. Antibiotics and antimycotic chemicals for culture of glochidia.

\begin{tabular}{lc}
\hline \hline \multicolumn{1}{c}{ Compound } & $\begin{array}{r}\text { Concentration } \\
\left(\mu \mathrm{g} \mathrm{L}^{-1}\right)\end{array}$ \\
\hline Antibiotics & 100 \\
Carbenicillin & 100 \\
Gentamycin sulfate & 100 \\
Rifampin & \\
Antimycotic & 5 \\
Amphotericin B & \\
\hline
\end{tabular}

achieved. The culture water was changed daily, by substituting half the culture volume from the same stock solution. Most juveniles came from the fishplasma culture medium and were fed for $15 \mathrm{~d}$.

\section{Morphological observations}

For microscopic studies, marsupia were dissected from gravid mussels, and the central part of each was cut to facilitate the visualization of glochidia within the brooding water tubes. Metamorphosis was observed using light microscopy; after metamorphosis, shell growth, as well as the appearance of gills and the accumulation of organic matter inside the gastrointestinal tract, was also observed. Juvenile activity was recorded by video, and some observations using SEM were carried out.

For SEM observations, sections $\sim 2-5 \mathrm{~mm}$ in width and 5-10 $\mathrm{mm}$ in length were fixed in $10 \%$ neutral buffered formalin for $2 \mathrm{~h}$ and preserved in $5 \%$ neutral buffered formalin for $24 \mathrm{~h}$. The specimens were then dehydrated in a graded series of ethanol and critical point dried. An identical procedure was used with both glochidial and juvenile samples. Before fixation, glochidia and juveniles were anesthetized in $2 \%$ chloral hydrate to observe the foot and mantle. The organic structures of some larvae were removed by bacterial decomposition for 1 week, and cleaned by distilled water to allow the observation of the internal calcareous layer of the shell. All samples were mounted on SEM specimen stubs with conductive carbon paint, coated with gold, and observed with a Jeol JSM-35 CF SEM (Jeol, France) operated at $25 \mathrm{kV}$. The assessment of hardening of new juvenile periostracum was based on relative calcium deposition analysis by energy-dispersive X-ray spectroscopy (EDS) (Jeol), with a spectrum collection time of $60 \mathrm{~s}$.

\section{Results}

\section{Marsupial gills and glochidia}

The marsupial gills of females of Anodonta cygnea are located in the outer demibranchs. The developmental appearance of marsupia coincided with the formation of secondary water tubes; the primary water tubes housed the embryos. The color of marsupia changed with stage of development, from transparent yellow with early embryos to dark brown before glochidial parturition. A fragment of marsupial gill from a gravid female with well-developed glochidia in the brood chamber is shown in Fig. 1. Gill filaments and an interlamellar septum can also be distinguished. The glochidia were released from 


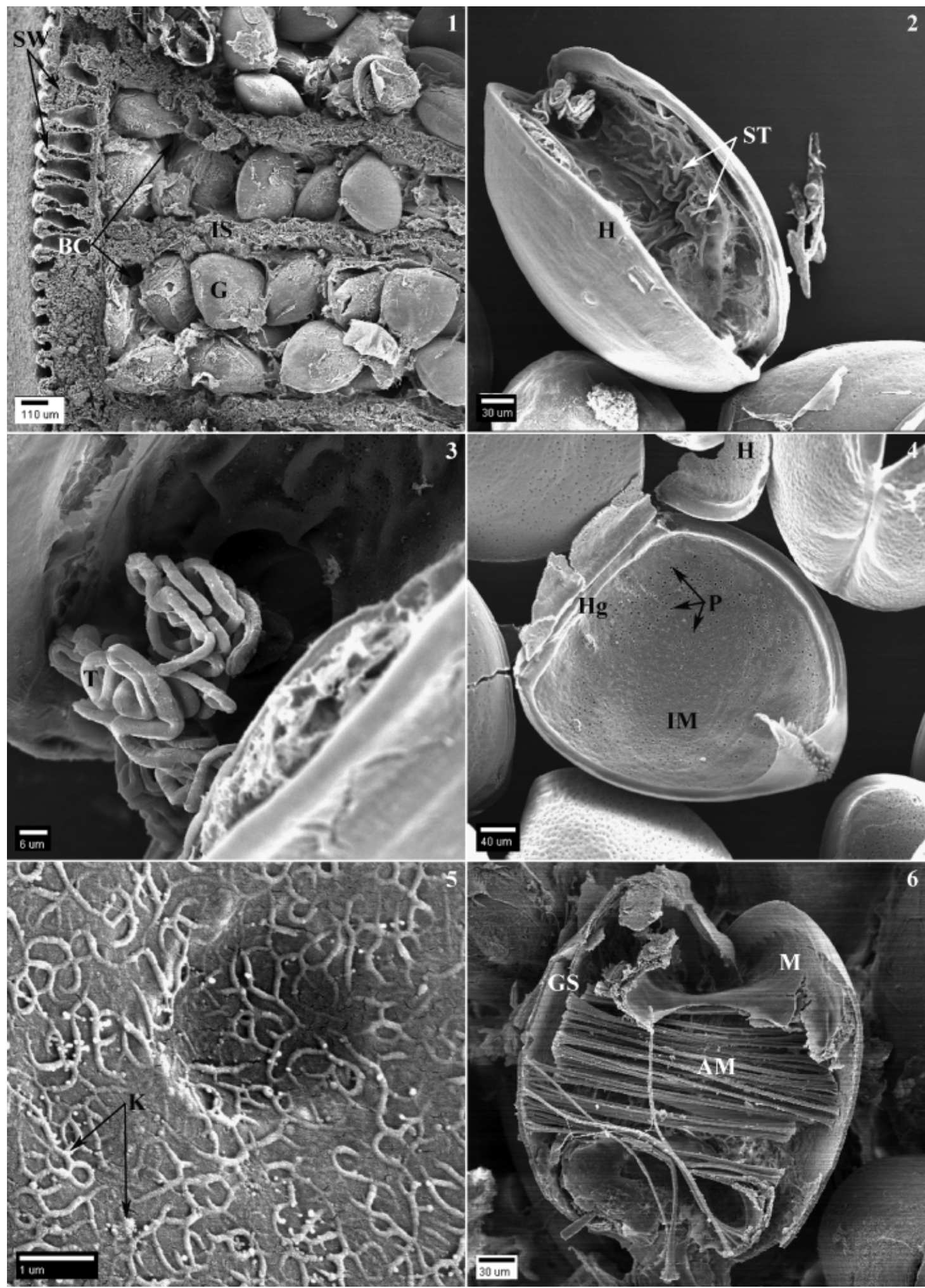

Figs. 1-6. Scanning electron microscopy of glochidia collected from gills of a gravid female. Fig. 1. Fragment of marsupial gill with glochidia $(\mathrm{G})$ in brood chamber $(\mathrm{BC})$; note secondary water tubes (SW) and interlamellar septum (IS). Fig. 2. Equi-valve shell of glochidia with hook $(\mathrm{H})$ covered with spines; note sensorial hair tufts. Fig. 3. Larval thread (T). Fig. 4. Shell hinge $(\mathrm{Hg})$ or glochidial shell valve; note pores in the internal calcareous layer, insertion area of adductor muscle (IM). Fig. 5. Keratin fibers surrounding one of the numerous pits in the external surface layer of the shell (K). Fig. 6. Broken glochidial shell showing the strong adductor muscle (AM) and fragments of the covering mantle (M). 
Table 3. Range and average percent survival and completed metamorphosis of glochidia in artificial media ( 30 replicates, 50-100 glochidia/culture).

\begin{tabular}{ccccc}
\hline \hline \multirow{2}{*}{ Media } & \multicolumn{2}{c}{ Survival } & & $\begin{array}{c}\text { Completed } \\
\text { metamorphosis }\end{array}$ \\
\cline { 2 - 5 } & Range (\%) & Mean (SD) & Range (\%) & Mean (SD) \\
\hline M199+fish plasma & $25.9 \pm 49.1$ & $34.3 \pm 9.3$ & $57.8 \pm 63.7$ & $60.8 \pm 4.2$ \\
\hline
\end{tabular}

brood chambers in clumps enveloped in mucus; they had triangular and equi-valve shells, with $\sim 350 \mu \mathrm{m}$ length and width and $180 \mu \mathrm{m}$ maximum thickness. Shells possess a strong and prominent hook in this species (Fig. 2). The glochidial thread used for attachment to the host fish was present (Fig. 3). The valves are joined by a straight hinge line $240 \mu \mathrm{m}$ in length, and the oval areas present in their internal surfaces correspond to insertion regions of the adductor muscle (Fig. 4). The internal surfaces of glochidial valves show numerous pores, whereas the external surface is covered with a fibrillar organic matrix (Figs. 4, 5). The valves are connected by a strong adductor muscle (Fig. 6), and a triplet of sensorial tufts is present in each valve (Fig. 7).

\section{Glochidia and juvenile culture}

Some aspects of metamorphosis occurred successfully within 9-10 d in the culture medium, e.g., the edge of definitive mantle outside the shell was evident. During metamorphosis, larvae remained triangular in shape until the foot appeared. We found that glochidia could successfully metamorphose into the juvenile stage in media containing fish plasma or horse serum as the protein source; however, as the preliminary culture experiments with horse serum achieved slightly poorer results compared with fish plasma, and noting the results of Uthaiwan et al. (2001a), we decided to culture glochidia only in fish plasma.

The complete period of metamorphosis was $10-11 \mathrm{~d}$. The average survival in fish plasma from glochidium to early juvenile stage $(\mathrm{p}<0.05)$ was $34.3 \pm 9.3 \%$ and the average rate of successful metamorphosis $(\mathrm{p}<0.05)$ was $60.8 \pm 4.2 \%$ (Table 3 ). Metamorphosis was observed under light microscopy until appearance of the foot, which is a clear indication of early juvenile survival and signals completion of metamorphosis (Figs. 8-15). The formation of the foot involves several growing stages from the two small symmetric and curved lobes in the region where the juvenile foot is located (Fig. 8). These lobes develop into larger and well-defined structures over a single solid base that grows upward (Figs. 9, 10).
Then the two distinct lobes fuse, forming a bifurcated structure until completely joined, to form a definitive foot covered by long cilia (Figs. 11-14). Figure 14 shows the primitive structures from which the internal and external gills form. The expanded foot and gills were clearly observed, as well as other important details of juvenile formation. The foot made quick movements when stimulated by the addition of distilled water and aeration (Fig. 15). The shell grew rapidly during the first days of juvenile life, with the original glochidial valves fixed near the hinge (Fig. 16). The growth lines on the juvenile shell were elongated.

Shell growth was more pronounced in the anterior region in 7-d-old juveniles (Figs. 17, 18). The new shell grows toward the hook, forming a slight depression in this area. Shell growth is marked by the addition of parallel, slender lines, initially in the anterior region. The juvenile shell is also hardened because of calcium deposition as shown by EDS analyses. These juveniles show an increase of $\sim 70 \mu \mathrm{m}$ in width of the new periostracum. The mantle bordering the new shell (Fig. 19) and the primitive gills are both covered with cilia. Finally, by $15 \mathrm{~d}$ of juvenile life (Fig. 20), a growth of $\sim 425 \mu \mathrm{m}$ in width of the new periostracum occurred, mainly in the lateral and posterior regions, also associated with a marked hardening by calcium deposition.

Complementary images were obtained of the in vivo condition by light microscopy (Figs. 21, 22). It was possible to see the mobile foot entirely covered with long cilia. The foot actively contracts and expands by muscular activity, reaching twice the size of the shell when valves are kept open. Growth lines on the juvenile shell are evident with a particular increase in the anterior region. The digestive tract is already formed in the juvenile stage, as seen through the shell.

\section{Discussion}

\section{Marsupial gills and glochidia}

In the present study, the expansion of the marsupial demibranch, caused by embryonic development 


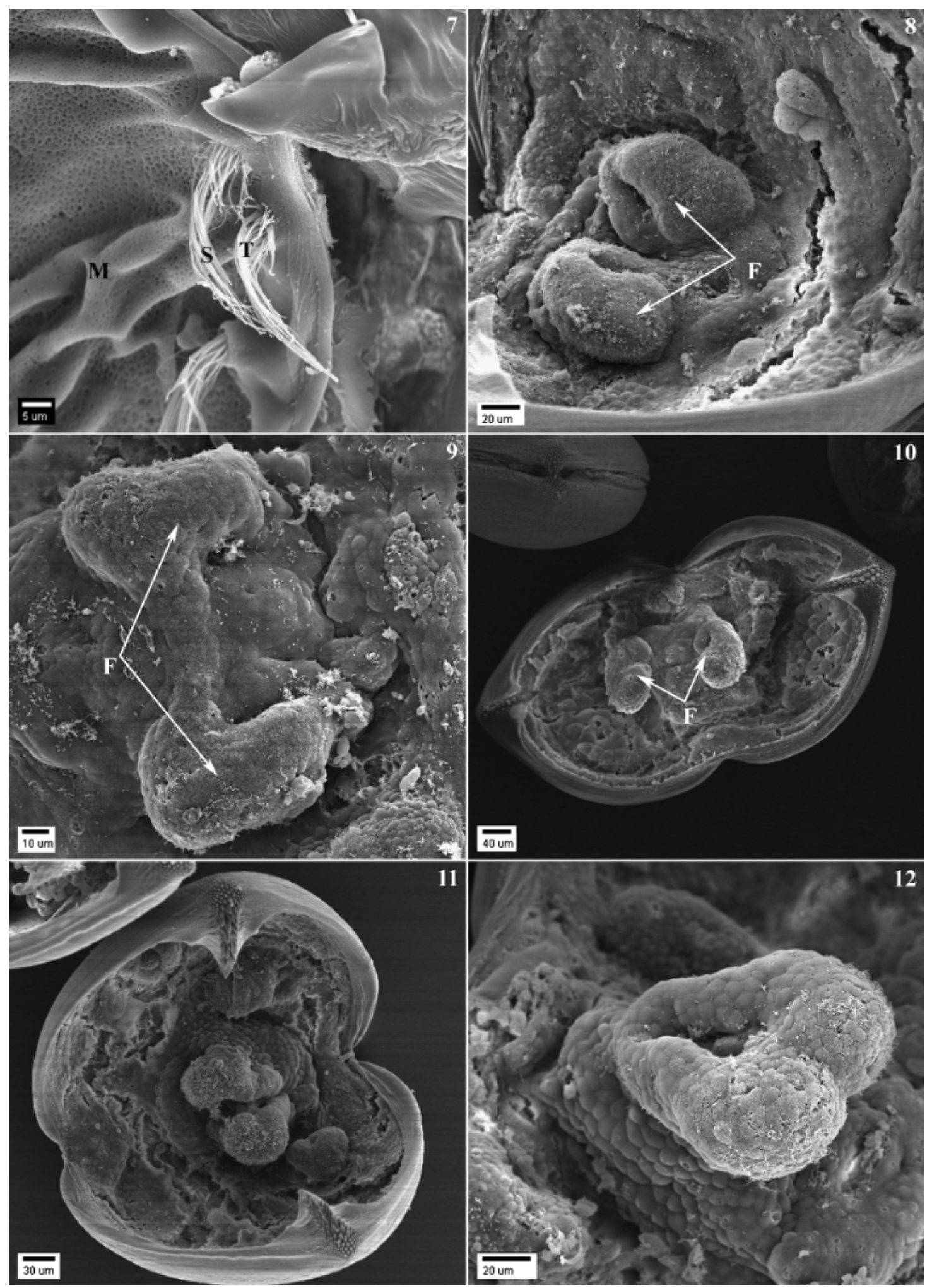

Figs. 7-12. Sensorial tufts (ST). Scanning electron microscopy of juveniles after $11 \mathrm{~d}$ of glochidial development, and additional periods of juvenile life. Figs. 8-12. Development stages of foot (F) organogenesis. 

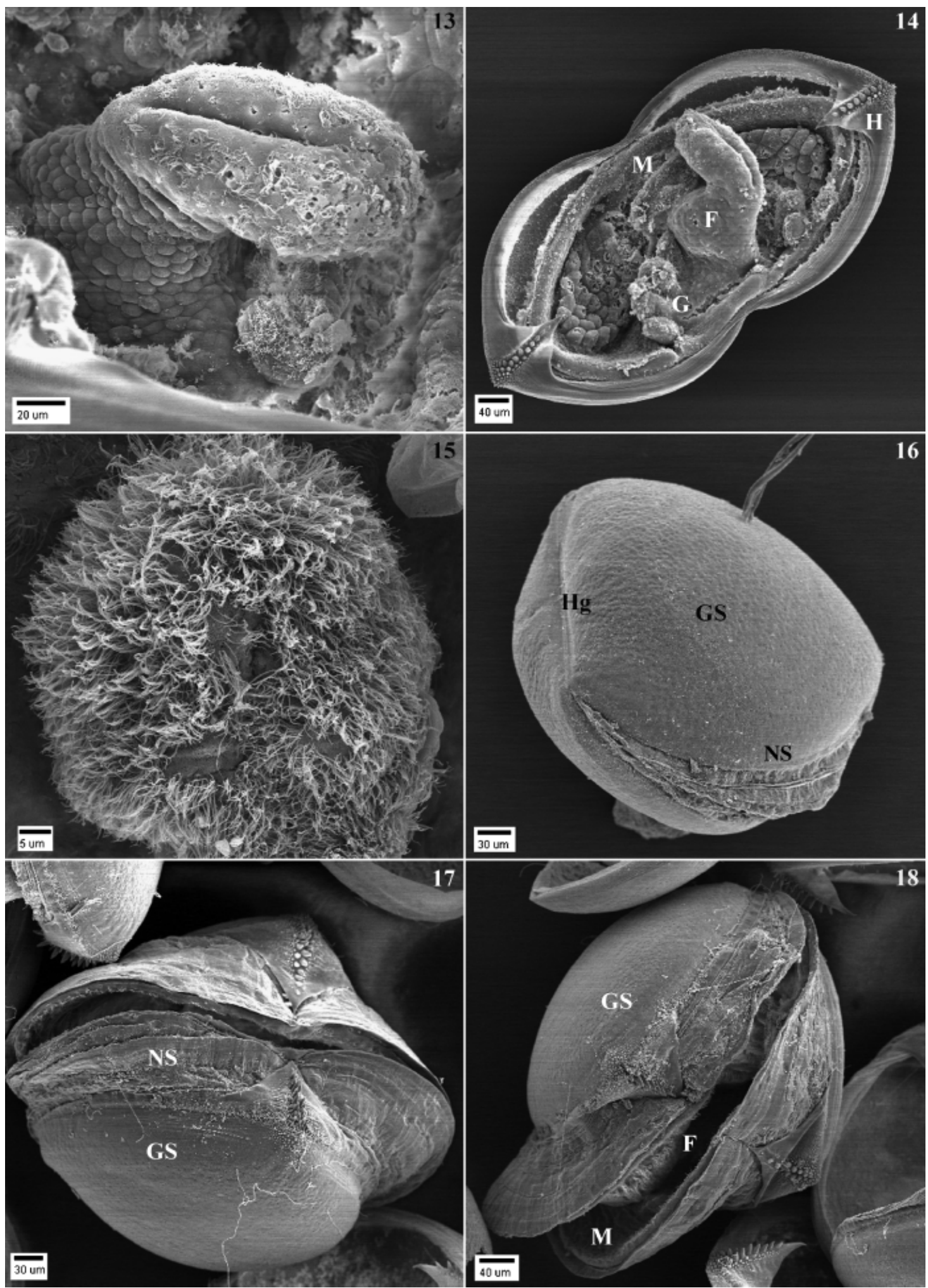

Figs. 13-18. Development stages of juvenile. Fig. 14. One-day-old juvenile; note foot (F), primitive gills (G), mantle (M), and hook (H). Fig. 15. Amplified foot (F) covered with cilia. Fig. 16. Three-day-old juvenile; note the appearance of new soft periostracum (NS) formed by organic matrix bands under the old cuticle of the glochidial shell (GS). Figs. 17-18. Seven-day-old juveniles; note new periostracum growth and its hardening by calcium deposition, internal mantle surface with numerous cilia. 


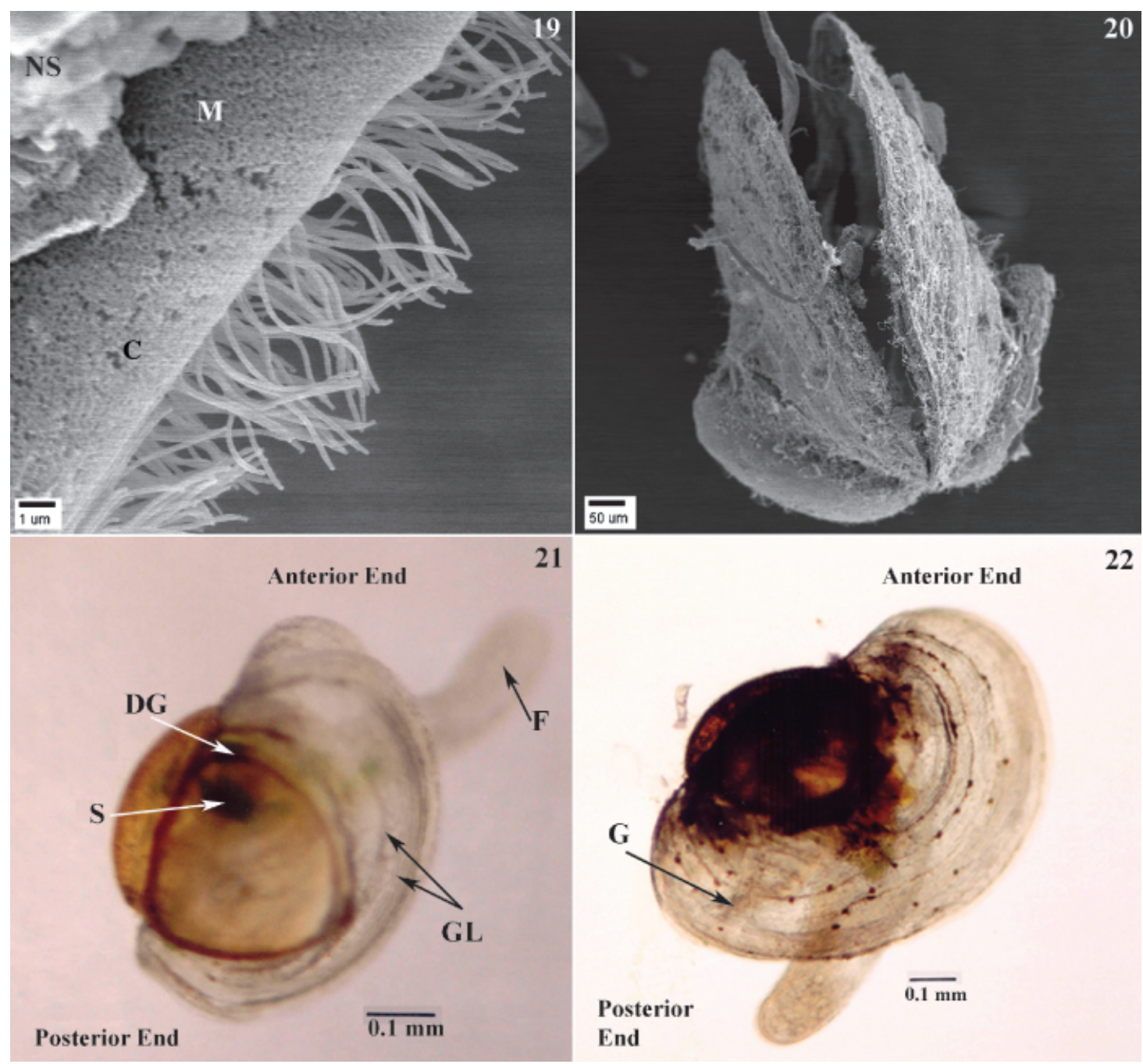

Figs. 19-22. Seven-day-old juveniles; note new periostracum growth and its hardening by calcium deposition, internal mantle surface with numerous cilia (C). Fig. 20. Fifteen-day-old juvenile shell; note the growth of juvenile periostracum. Light photomicrographs of juveniles after $11 \mathrm{~d}$ of glochidial development, and additional periods of juvenile life. Fig. 21. Ten-day-old juvenile; note that the foot (F), stomach (S), and digestive gland (DG) are visible under the thin shell. Fig. 22. Thirteen-day-old juvenile; note new periostracal growth marked by additional growth lines (GL) in the shell; foot (F) and gills (G) are visible.

and glochidia incubation over 2-3 months, was observed. According to Smith (1979), this marsupial incubation has been considered an advanced feature in freshwater mussels. Glochidia shells of Anodonta cygnea showed a triangular valve shape, with a hook from the incubation period in brood chambers onward. Numerous scattered pits, resulting from the presence of an organic pellicle over the holes in the internal layer of the valves, were observed. It was also observed that the organic pellicle of the shell covers an internal calcareous layer of calcium carbonate crystals. In the ventral region, we observed a hook and many spines around the shell border and a larval thread extending between the valves. The mantle presented a few sensory hair cells and an adductor muscle responsible for shell closure.

These observations show that the glochidial shell of $A$. cygnea has several ultrastructural aspects similar to those described previously in glochidia of Margaritifera, Unio, and Anodonta (Lillie 1895; Herbers 1914; Wood 1974; Castilho et al. 1989; Nezlin et al. 1994; Pekkarinen \& Englund 1995b; Pekkarien 1996; Pekkarinen \& Valovirta 1996, 1997; Araújo \& Ramos 1998; Hoggarth 1999), such as a hook, spines, internal pores, external pits, cilia, an adductor mussel closing and opening the valves, 
and an internal calcareous layer. The external organization of the cuticle shows the presence of keratin fibers with parallel arrangement and embedded in a chitin matrix as described by Castilho et al. (1989).

\section{Glochidia and juvenile culture}

In this study, metamorphosis was induced in artificial culture without pollutant factors, producing the early juvenile stage within $10-11 \mathrm{~d}$. Additional data from microscopic observations gave good morphological information on the development of early juveniles. The foot, gills, gastrointestinal tract, shell growth lines, and movements are obvious signs of complete metamorphosis.

Our results showed that the survivorship rate was $34.3 \pm 9.3 \%$ and the proportion of survivors completing metamorphosis was $60.8 \pm 4.2 \%$ in M199 medium (Cyprinus carpio). The rate of metamorphosis in fish plasma was lower than the results from similar experiments $(81.8 \pm 7.5 \%)$ in the Isom \& Hudson medium performed by Keller \& Zam (1990). However, the current results are significant in that the period of metamorphosis for glochidia cultured in fish plasma showed less variation, and the medium is more available (M199). This is also the first time that any results were obtained in European specimens. The success of the artificial medium can still be improved, considering that host specificity is a crucial factor to development, given the physiological adaptations of glochidia as discussed by Yeager \& Saylor (1995) and Uthaiwan et al. (2002, 2003).

The rate of completed metamorphosis, in the Isom \& Hudson medium with fish plasma, by Keller \& Zam (1990) was high but resulted in inactive and lethargic juveniles. On the contrary, the artificial medium (M199; DME) with horse serum by Keller \& Zam (1990) produced a significantly lower proportion of completed metamorphosis but healthier individuals. On the other hand, Uthaiwan et al. (2001a,b, 2002) improved this medium formula and showed that the combination of artificial media (M119) and fish plasma can restore the high percentage of individuals completing metamorphosis and a high survivorship during juvenile stages until 3 months of age. We used this formula in our experiments, and also obtained healthy and active juveniles. However, our juveniles survived only $15 \mathrm{~d}$; therefore, culture conditions can be improved.

Many selective factors influence the success of juvenile development after transformation. As glochidia are easier to find, their morphology is better known than that of juvenile stages. Little attention has previously been paid to morphological develop- ment during early juvenile stages (D'Eliscu 1972). However, a few studies of late juvenile development have been carried out (Harms 1907, 1909; Herbers 1914). Certainly, a controlled process involving the artificial culture of glochidia facilitates observations from metamorphosis to juvenile stages. In vitro culture was difficult to accomplish until the methods of Keller \& Zam (1990) and Uthaiwan et al. (2001a) substantially improved glochidial survival and development. Therefore, the main objective of the present work was not only to find the ideal conditions for successful development to the juvenile stage, using the method by Uthaiwan et al. (2001a), but also to provide detailed morphological descriptions of the early juvenile stage as well as characteristics indicative of completed metamorphosis.

We have now shown that, in A. cygnea, the beginning of juvenile shell growth occurs mainly in the anterior region by the formation of a soft periostracum during the first $3 \mathrm{~d}$ of juvenile life. Partial hardening of the periostracum because of calcium deposition was observed at $5-8 \mathrm{~d}$, followed by a marked hardening at $13 \mathrm{~d}$ of juvenile life in the lateral and posterior regions of the new periostracum. Simultaneously, regression of the old cuticle of the glochidial shell is also apparent at this stage. A characteristic of juvenile behavior is the continuous opening and closing of the valves and continual movements of the prominent foot in and out of the intrapallial cavity. A particular aspect of foot organogenesis is related to a pair of primitive lobes on the base of the future foot, which will gradually grow first as a long bifurcated structure and finally as a single one. In our opinion, this interesting aspect of foot organogenesis is supported by the fact that bivalves show a bilaterally symmetrical development of paired gonads, which will be included inside the entire foot (Moore 1983).

The presence of long cilia on the foot may indicate a role in feeding by the capture of small organic particles. Smaller cilia in the mantle epithelium may also have this function. The microvilli on the external surface of the mantle of juveniles, similar to those observed in the adult mantle of A. cygnea (Machado et al. 1988), may well be involved in the absorption of organic and inorganic molecules dissolved in the water. Longer cilia, sparsely distributed in the mantle epithelium of juveniles, may correspond to the sensory hair tufts in glochidia as observed by Wood (1974), Pekkarinen \& Englund (1995a), and Pekkarinen \& Valovirta (1996). According to these authors, the hair cells may be chemosensory or transmit tactile stimuli by the hairs, inducing shell closure.

It is possible that, in artificial culture, survival and metamorphic percentages in A. cygnea may be 
improved through the study of plasma specificity of European fish, as Uthaiwan et al. (2002) accomplished for tropical fish species. This means that survival of glochidia depends fundamentally on the appropriate fish species as a specific plasma source. Parallel studies on fish infestation by glochidia could give additional valuable information, which could be applied to the artificial cultures of $A$. cygnea. Future research should also focus on the diets of juvenile stages during the very delicate period during which the digestive tract is not yet adapted to solid food. Because we induce metamorphosis at higher temperatures than occur in nature, organogenesis is accelerated and the new organism may respond normally to physiological demands or immunological defence mechanisms. In fact, Fisher \& Dimock (2002) stated that larvae reared on a host fish accumulated substantial deposits of lipids and glycogen within larval mantle cells during metamorphosis, whereas larvae reared in vitro did not.

Finally, although juveniles produced by incubation in artificial medium seem to be less healthy than those produced by infestation, we think that this method has potential value as high percentages of juveniles can be achieved. Further studies of these methods will include comparative studies of naturally produced versus artificially cultured juveniles.

Acknowledgments. We are very thankful to Dr. Pintip Karnasuta, Head of the Zoology Department of the Science Faculty, Kasetsart University, for permitting us to develop our research in the zoology lab. We thank Dr. Jorge Sequeiros for allowing us to use specific equipment from his lab, in the Medical Genetics Centre, IBMC. We also thank Mr. Andrade for helping us with blood collection and Dr. Jonathan Mark Wilson for the English corrections. This work was supported by the Portuguese Science and Technology Foundation (FCT).

\section{References}

Araújo R \& Ramos MA 1998. Description of the glochidium of Margaritifera auricularia (Bivalvia, Unionoidea). Phil. Trans. R. Soc. Lond. 353B: 1553-1559.

Arey LB 1932. A microscopical study of glochidial immunity. J. Morphol. 53: 367-379.

Castilho E, Machado J, Reis ML, \& Sá C 1989. Utrastructure study of the embryonic and larval shell of Anodonta cygnea. Can. J. Zool. 67: 1659-1664.

D'Eliscu PD 1972. Observation of the glochidium, metamorphosis, and juvenile of Anodonta californiensis (Lea, 1857). Veliger 15: 57-59.

Fisher GR \& Dimock RV 2002. Ultrastructure of the mushroom body: digestion during metamorphosis of Utterbackia imbecillis (Bivalvia: Unionidae). Invert. Biol. 121: 126-135.
Haag WR \& Warren ML 1999. Mantle displays of freshwater mussels elicit attacks from fish. Freshwater Biol. 42: $35-40$.

2000. Effects of light and presence of fish on lure display and larval release behaviours in two species of freshwater mussels. Anim. Behav. 60 (6): 879-886.

Harms W 1907. Uber die postembryonale Entwicklung von Anodonta piscinalis. Zool. Anz. 31: 801-807.

1909. Postembryonable Entwicklungsgeschichte der Unioniden. Zool. Jahrb. Abt. Anatomie Ontog. Tiere 28: 326-386.

Herbers K 1914. Entwicklungsgeschichte von Anodonta cellensis Schröt. Zeitscr. Wiss. Zool. 108. 174 pp.

Hoggarth MA 1999. Descriptions of the glochidia of the Unionidae (Mollusca: Bivalvia). Malacologia 41 (1): $1-118$.

Howells RG, Neck RW, \& Murray HD 1996. On Freshwater Mussels of Texas. Parksand Wildlife Press, Austin, TX. 218 pp.

Isom BG \& Hudson RG 1982. In vitro culture of parasitic freshwater mussel glochidia. Nautilus 96: 147-151.

1984a. Freshwater mussels and their fish hosts; physiological aspects. J. Parasitol. 70 (2): 318-319.

1984b. Culture of freshwater mussel glochidia in an artificial habitat utilizing complex liquid growth media. US Patent 4,449,480. 18 pp.

Jokela J \& Palokangas P 1993. Reproductive tactics in Anodonta clams: parental host recognition. Anim. Behav. 46: 618-620.

Keller AE \& Zam SG 1990. Simplification of in vitro culture techniques for freshwater mussels. Environ. Toxicol. Chem. 9: 1291-1296.

Kirk SG \& Layzer JB 1997. Induced metamorphosis of freshwater mussel glochidia on nonhost fish. Nautilus 110 (3): 102-106.

Lillie FR 1895. The embryology of the Unionidae: a study in cell-lineage. J. Morphol. 10: 1-100.

Machado J, Castilho F, Coimbra J, Monteiro E, Sa C, \& Reis M 1988. Ultrastructural and cytochemical studies in the mantle of Anodonta cygnea. Tiss. Cell 20: 797-807.

Mansur MC \& Silva MG 1999. Description of glochidia of five species of freshwater mussels (Hyriidae: Unionoidea) from South America. Malacologia 41 (2): 475-483.

Meyers TR \& Millemann RE 1977. Glochidiosis of salmonid fishes. I. Comparative susceptibility to experimental infection with Margaritifera mararitifera (L.) (Pelecypoda: Margaritanidae). J. Parasitol. 63: 728-733.

Meyers TR, Millemann RE, \& Fustish CA 1980. Glochidiosis of salmonid fishes. IV. Humoral and tissue responses of coho and chinook salmon to experimental infection with Margaritifera. J. Parasitol. 66: 274-281.

Moore B 1983. Organogenesis. In: The Mollusca, Vol. 3. Development. Verdonk N, Biggelaar JA, \& Tompa A, eds., pp. 123-177. Academic Press, New York.

Nagachinta A \& Meejui O 1998. Reproductive biology of the Thai freshwater pearl mussel Hyriopsis (Limnoscapha) myersiana (Lea, 1856). Phuket Marine Biological Center Special Publication 18: 103-106. 
Nagachinta A, Sanassanonta N, Tispijit K, \& Tispijit N 1986. Preliminary study on pearl culture in two species of freshwater mussel. In: Proceedings of the Annual Meeting Seminar. pp. 395-401. Kasetsart University, Fisheries Section, Bangkok.

Nezlin LP, Cunjak RA, Zotin AA, \& Ziuganov VV 1994. Glochidium morphology of the freshwater pearl mussel (Margaritifera margaritifera) and glochidiosis of the Atlantic salmon (Salmo salar): a study by scanning electron microscopy. Can. J. Zool. 72: 15-21.

O'Connell MT \& Neves RJ 1999. Evidence of immunological responses by a host fish (Ambloplites rupestris) and two non-host fishes (Cyprinus carpio and Carassius auratus) to glochidia of a freshwater mussel (Villosa iris). J. Freshwater Ecol. 14 (1): 71-78.

Panha S \& Kosavitikul P 1977. Mantle transplantations in freshwater mussel in Thailand. Aquaculture 5: 267-276.

Pekkarien M 1996. Scanning electron microscopy, whole mount histology, and histochemistry of two anodontine glochidia (Bivalvia: Unionidae). Can. J. Zool. 74: 1964-1973.

Pekkarinen M \& Englund VPM 1995a. Sizes of intramarsupia unionacean glochidia in Finland. Arch. Hydrobiol. 134: 379-391.

1995b. Description of unionacean glochidia in Finland, with a table aiding in their identification. Arch. Hydrobiol. 134: 515-531.

Pekkarinen M \& Valovirta I 1996. Anatomy of the glochidia of the freshwater pearl mussel, Margaritifera margaritifera (L.). Arch. Hydrobiol. 137: 411-423.

1997. Histochemical and X-ray studies on tissue concretions and shells of Margaritifera margaritifera (Linnaeus). J. Shellfish Res. 16: 169-177.
Pennak RW 1978. Freshwater Invertebrates of the United States, 2nd ed. John Wiley, London. 628 pp.

Reuling FH 1919. Acquired immunity to an animal parasite. J. Infect. Dis. 24: 337-346.

Rogers SO, Watson BT, \& Neves RJ 2001. Life history and population biology of the endangered tan riffleshell (Epiblasma florentina walkeri) (Bivalvia: Unionidae). J. N. Am. Benthol. Soc. 20 (4): 582-594.

Smith DG 1979. Marsupial anatomy of the demibranch of Margaritifera margaritifera (Lin.) in northeastern North America (Pelecypoda: Unionacea). J. Moll. Stud. 45: 39-44.

Uthaiwan K, Noparatnaraporn N, \& Machado J 2001a. Culture of glochidia of the freshwater pearl mussel Hyriopsis myersiana (Lea, 1856) in artificial media. Aquaculture 195 (1-2): 61-69.

$2001 \mathrm{~b}$. Scanning electron microscopy of glochidia and juveniles of the freshwater mussel, Hyriopsis myersiana. Invertebr. Reprod. 40 (2-3): 143-151.

Uthaiwan K, Pakkong P, Noparatnaraporn N, Vilarinho L, \& Machado J 2002. Study of a suitable fish plasma for in vitro culture of glochidia Hyriopsis myersiana. Aquaculture 209 (1-4): 197-208.

2003. Studies on the plasma composition of host fish: implications on the culture medium improvement for growth of Hyriopsis myersiana. Invertebr. Reprod. 44 (1): 53-61.

Wood EM 1974. Development and morphology of the glochidium larvae of Anodonta cygnea (Mollusca: Bivalvia). J. Zool. Lond. 173: 1-13.

Yeager B \& Saylor CF 1995. Fish hosts for four species of freshwater mussels (Pelecypoda: Unionidae) in the upper Tennessee River Drainage. Am. Midl. Nat 133 (1): 1-6. 